
et dans les oeuvres de ses contemporains

\title{
Le déni de justice dans The Spanish Tragedy: Hiéronimo, juge et justicier
}

\section{Jean Fuzier}

Marie-Thérèse Jones-Davies (éd.)

\section{(2) OpenEdition}

\section{Journals}

\section{Édition électronique}

URL : http://journals.openedition.org/shakespeare/126

DOI : $10.4000 /$ shakespeare. 126

ISSN : 2271-6424

Éditeur

Société Française Shakespeare

Édition imprimée

Date de publication : 1 novembre 1980

Pagination : 85-98

Référence électronique

Jean Fuzier, «Le déni de justice dans The Spanish Tragedy: Hiéronimo, juge et justicier », Actes des congrès de la Société française Shakespeare [En ligne], 2 | 1980, mis en ligne le 01 novembre 2007, consulté le 23 avril 2019. URL : http://journals.openedition.org/shakespeare/126 ; DOI : 10.4000/ shakespeare.126 


\section{SOCIÉTÉ FRANC̣AISE SHAKESPEARE}

\section{ACTES DU CONGRĖS 1980}

DIRECTEUR DE LA PUBLICATION

M.T. Jones - Davies

JEAN TOUZOT Libraire - Editeur 38 , rue Saint-Sulpice 75278 PARIS CEDEX 061981 


\section{LE DENI DE JUSTICE DANS THE SPANISH TRAGEDY DE THOMAS KYD : HIERONIMO, JUGE ET JUSTICIER}

Il ne s'agit pas ici de faire des révélations capitales à propos d'une œuvre déjà fort étudiée, quoique souvent sousestimée, mais simplement de procéder à une réévaluation circonstanciée de son importance et de son originalité par rapport au thème des juges et de la justice qui est celui de notre colloque, autant que par rapport aux cuvres ultérieurs qui, du Hamlet de Shakespeare au Cardinal de Shirley, n'auraient sans doute jamais vu le jour sans The Spanish Tragedy. Toutes ces pièces, au nombre d'une soixantaine, qui furent écrites et jouées entre 1587 et 1641 , et qui constituent le corpus de la tragédie de vengeance commodément appelée élisabéthaine même si, historiquement, elle est également jacobéenne et caroléenne, doivent en effet, directement ou indirectement, quelque chose à Kyd,qu'il $s^{\prime}$ agisse du fantôme, de la folie, du nocturne, du théâtre dans le théâtre ou, bien sur, de la vengeance qui apparaît, abusivement peutetre, comme leur dénominateur commun. Kyd n'a pas inventé le fantôme tragique, bien que, tout en le confinant dans un rôle en apparence extérieur limité à la pièce d'encadrement, il ait su lui donner une valeur à la fois structurale et fonctionnelle dans le mécanisme de la vengeance, voire une dimension à la fois éthique et religieuse que n'avaient ni le fantôme de Tantale, ni même celui d'Agrippine,dans le Thyeste ou l'Octavie de Sénèque. Il n'a pas inventé la folie qui, d'Oreste à Hercule et d'Ajax à Penthée, est un ressort tragique connu de l'antiquité classique : mais il a su l'associer à la vengeance en lui attribuant une valeur simultanée d'excuse et de masque plutôt que de châtiment divin, ce qui était sa fonction antique. Il n'a pas inventé non plus le nocturne théâtral, mais a du moins su exploiter la somme de ses possibilités pour l'accomplissement du crime et de la vengeance, lui donnant son statut tragique, qui ne pourra qu'être confirmé par ses imitateurs et successeurs ${ }^{1}$. Il a par contre, on peut le dire,inventé le théâtre dans le théâtre sous sa forme la plus authentique, et l'a lié à la vengeance de façon telle que ses descendants ne parviendront pas à s'en dégager ${ }^{2}$. Et, s'il n'a pas inventé la vengeance en tant que 
motivation tragique, il a du moins mis au point (et c'est là son mérite le plus incontestable) une formule dramatique associant à la vengeance les quatre éléments (fantôme, folie, nocturne, théâtre dans le thêâtre) que je viens de citer, formule que ses nombreux successeurs tâcheront en vain pendant plus de cinquante ans, dans des œuvres d'une qualité dramatique ou littéraire pourtant mainte fois supérieure, d'imiter, d'égaler, ou d'améliorer.

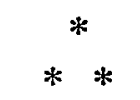

Cela dit, je me propose de montrer ou de démontrer ici que The Spanish Tragedy, si elle est sans conteste la mère ou le prototype de la tragédie de vengeance élisabéthaine, ne l'est peut-être pas, en fin de compte, sur les bases les plus légitimes, car à l'examen elle ne se révèle pas tellement comme une tragédie de vengeance que comme la tragédie par excellence de la justice, justice que l'on y trouve présente à tous ses échelons, de l'humain au divin, en passant par le royal. Je soulignerai d'abord, car ceci a son importance dans la mesure où la pièce de Kyd évolue de façon parallèle, pour ce qui est de la vengeance et de la justice, sur le plan divin et sur le plan humain,l'extrême ambiguïté de la notion biblique de vengeance, ce terme étant une des appellations les plus courantes de la justice divine, plus répandu peut-être en ce sens, dans l'Ancien Testament, que le mot «justice» lui-même ${ }^{3}$. Le code mosaïque prévoit d'autre part des cas, rares il est vrai, mais bien précis, où la vengeance individuelle est l'instrument élu de la vengeance (entendons de la justice) divine : le crime de sang dont un parent proche a été la victime est de ceux-là ${ }^{4}$. Ce préambule n'est pas destiné à justifier, en vertu des anciennes lois hébraïques, des procédés que condamne la législation élisabéthaine; son but est simplement de bien marquer que justice et vengeance sont deux notions qui, pour un Anglais de la fin du XVIe siècle nourri des Ecritures, présentent une importance zone de recoupement à la fois sémantique et pragmatique, et que, si tant est que la justice est vengeance au niveau suprême, on peut inversement concevoir que la vengeance soit justice au degré le plus bas dans la mesure où les textes sacrés couvrent de leur autorité cette confusion des termes. 
Kyd n'a d'ailleurs pas recours à ce code de justice d'un autre âge (même si tout plaide afin qu'il soit pris en considération) pour justifier les actes d'Hiéronimo, dont la vengeance nous est présentée dans la pièce, par divers moyens et grâce à diverses précautions liminaires, comme un acte de justice légitimé à ses différents stades, même si le juge peut sembler appelé par les circonstances à se transformer à un moment en justicier. Je dis bien justicier, et non vengeur, car la terminologie a ici son importance. L'acte ultime du vengeur est foncièrement privé, car il n'est animé que par des raisons d'ordre personnel, tandis que celui du justicier, même s'il peut avoir, comme c'est ici le cas, des motivations individuelles, se réclame d'un code, qu'il soit social, éthique, ou religieux. Etre vengeur, c'est appliquer pour son propre compte la loi biblique du talion sous sa forme la plus simpliste, en prenant oil pour œil, dent pour dent et sang pour sang, sans considérer un seul instant que cette loi a d'abord un fondement social et religieux, et que son application devrait donc dépendre du jugement et de l'agrément de la communauté à laquelle on appartient; c'est d'ailleurs aussi, parfois, prendre œil pour dent et sang pour œil, ou faire retomber sur des innocents le châtiment du ou des coupables. Etre justicier suppose au contraire, quelques motivations privées que l'on puisse avoir, le sens aigu d'une mission sociale ou religieuse, qui est de rétablir la justice là où elle a cessé de régner parce qu'elle est bafouée par l'autorité pour laquelle son exercice est un devoir autant qu'un droit. Le justicier n'agit qu'en accord avec sa conscience, et une partie au moins du corps social dont il est membre : corps social qui, dans le cas de situations fictives comme en présentent le théâtre et le cinéma (je pense ici à l'éthique du «western», version moderne de la tragédie de vengeance élisabéthaine, et genre, comme elle, éminemment populaire), est bien évidemment figuré par les spectateurs. La situation imaginée par Kyd dans The Spanish Tragedy me paraît à la fois beaucoup plus complexe et rigoureuse qu'on ne la considère en général, et certains problèmes y sont posés avec une clarté et une acuité que ne devait égaler aucun de ses successeurs. Cette situation fait intervenir une «vengeance» humaine comme instrument inconscient d'une «vengeance» divine, qui en fournit a.posteriori la justification, et en garantit la justice (Hiéronimo, 
en vengeant la mort de son fils Horatio, punit en fait, par décision divine, les coupables de la mort d'Andréa, dont le fantôme, escorté de la Vengeance, préside au déroulement de l'action). Mais c'est là une vision globale et extérieure des événements à laquelle on ne saurait se référer d"emblée, et qui ne peut intervenir de façon valable que comme lecture finale de la pièce. L'auteur, s'il joue également sur le plan divin et sur le plan humain, s'attache en effet à ne rien laisser au hasard pour que, dans toutes ses étapes, la démarche du vieil Hiéronimo suive la voie, ici paradoxalement ardue et tortueuse, de la justice, plutôt que le droit chemin de la simple vengeance personnelle. The Spanish Tragedy pose à mon avis avec une grande netteté, et une non moins grande habileté, un double problème, à la fois juridique et humain, que l'interférence des deux éléments rend particulièrement difficile à résoudre. C'est d'un double problème d'école qu'il s'agit, et qui n'aurait sans doute pu être mieux formulé si Kyd avait étudié aux Inns of Court, ce que sa biographie, pour fragmentaire qu'elle soit, ne permet pourtant pas de supposer. Et c'est à ce double problème que The Spanish Tragedy doit à la fois son intérêt essentiel et son originalité, tant par rapport à la tradition classique dont elle est née que par rapport à l'abondante postérité à laquelle elle a donné naissance.

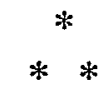

Pour bien comprendre cette situation et essayer de la résoudre, il est bon de rappeler d'abord quelques faits. Hiéronimo n'est pas, dans la pièce, que l'Intendant des Menus Plaisirs de la Maison du Roi d'Espagne, l'organisateur des spectacles de la Cour, rôle auquel il excelle d'ailleurs, et qui lui permettra d'atteindre ses fins lorsque tout autre mojen aura échoué. En tant que Maréchal de la Maison du Roi, il se voit aussi investi par Kyd, comme le fait très pertinement remarquer S.F. Johnson ${ }^{5}$, des prérogatives judiciaires du Knight Marshal of the Royal Household, qui avait en Angleterre «judicial cognizance of transgressions within the King's house and verge - i.e. within a radius of twelve miles from the King's palace.». Hiéronimo est donc le juge de la Cour, de la façon la plus officielle qui soit, et Kyd 
prend grand soin de nous le montrer dans l'exercice de ses fonctions lors de la condamnation de Pedringano à la pendaison pour le meurtre de Serberine, agencé par Lorenzo, meurtre pour lequel il y a eu flagrant délit, et qui a été commis près du palais royal ${ }^{6}$. Cette fonction judiciaire n'est d'ailleurs pas seulement une fonction accidentelle liée à sa charge, car Kyd précise d'autre part (III. xiii . 51-4) que le Maréchal est un juriste averti, le meilleur sans doute du pays, et un homme épris de justice. C'est du moins ainsi que le présentent les «petitioners» venus lui demander de plaider leur cause auprès du roi :

So I tell you this, for learning and for law, There's not any advocate in Spain

That can prevail, or will take half the pain That he will, in pursuit of equity.

Ceci posé, et bien posé, par Kyd, nous nous trouvons donc en présence d'un juge officiel autant que compétent confronté à une situation professionnelle et personnelle des plus délicates. La lettre trouvée par le bourreau dans les vêtements de Pedringano, et qui dénonce la part prise par Lorenzo et Balthazar au meurtre de son fils Horatio, le confirme en effet, selon le principe, biblique d'abord, mais adopté ensuite par de nombreuses jurisprudences : «testis unus, testis nullus ${ }^{7}$,» dans les soupçons qu'avait pu éveiller en lui le «bloody writ» de Belimpéria, cette note «in red ink», c'est-à-dire écrite de son propre sang, qu'elle avait jetée de la fenêtre de la chambre où son frère Lorenzo l'avait emprisonnée pour un temps après le meurtre d'Horatio dont elle avait été témoin. On ne saurait trop insister sur le caractère légaliste de ce double témoignage qui, aux yeux d'Hiéronimo, apparaît comme la condition déterminante de son passage à l'action, après une longue période d'indécision où sa raison vacille : cela d'autant plus que Kyd a pris soin de nous donner auparavant avec beaucoup d'insistance, l'exemple à ne pas suivre d'une erreur judiciaire flagrante, et réparée d'extrême justesse, dans le cas de la condamnation d'Alexandro par le vice-roi de Portugal sur le seul témoignage, mensonger, de l'envieux Villuppo ${ }^{8}$. La conviction du Maréchal est maintenant faite en toute légitimité, mais il se trouve alors doublement paralysé dans sa capacité officielle de juge, d'abord par le principe auquel, en tant que juriste, il ne saurait passer outre, à savoir que l'on ne peut être à la 
fois juge et partie, ensuite par le rang social des deux criminels survivants, qui échappent de toute manière à sa juridiction : il s'agit en effet du propre neveu du roi, Lorenzo, et du futur détenteur de la couronne, Balthazar, prince de Portugal, que son prochain mariage avec Bélimpéria doit faire prince héritier du trône d'Espagne. Et c'est donc, une nouvelle fois, une solution légaliste à l'extrême que choisit' Hiéronimo, faute qu'il puisse, en son âme et conscience, passer sentence sur d'aussi grands personnages dont le crime a bien été commis à l'intérieur des limites territoriales où s'exerce sa juridiction, mais sur la personne de son propre fils. Au lieu que le passage à l'action se traduise pour lui par le recours immédiat à la vengeance privée, c'est vers l'instance judiciaire supérieure qu'il décide naturellement de se tourner, c'est-à-dire la justice royale, seule habilitée en ce monde à statuer sur l'affaire.

Ici interviennent alors deux obstacles, qui n'ont rien de légal, mais il faut bien faire rebondir l'action dramatique. Le premier est Lorenzo, qui écarte physiquement Hiéronimo d'un roi d'ailleurs peu disposé à l'entendre au moment où il fait appel à lui, car il traite alors d'importants problèmes dynastiques :

Hier. Justice, O justice to Hieronimo!

Lor. Back! seest thou not the king is busy?

Hier. Oh, is he so?

King. Who is he that interrupts our business?

(III. xii. 27-30)

Le second est la défaillance de sa raison, qui l'empêche de mener à bien une nouvelle tentative, d'ailleurs derechef contrée par Lorenzo (III.xii. 59sqq.)., Le vieillard perd alors le contrôle de lui-même et de ses propos, ce qui lui ôte toute chance de se faire entendre, mais il conserve du moins assez de logique pour donner sa démission en tant que Maréchal, c'est-à-dire aussi bien en tant que juge, en ce monde dont la justice est désormais absente :

And here surrender up my marshalship (III.xii. 76). Est-ce là le signe que le magistrat va se muer en vengeur? On peut se le demander.... L'imagination de Kyd a cependant prévu de nouveaux rebondissements : la décision d'Hiéronimo est mise par le roi, encouragé en cela par Lorenzo, sur le compte d'une folie dont les raisons réelles ne sont évidemment pas évoquées : le roi ignorera en effet jusqu'au dénoue- 
ment la véritale cause de la disparition d'Horatio, et de la démence de son père. Il refuse ainsi, malgré les pressions de Lorenzo, d'accepter la démission du Maréchal, et la balance continue donc de pencher du côté de la justice, car c'est toujours officiellement investi de son pouvoir judiciaire qu'Hiéronimo, juge-justicier, exécutera sa «vengeance». Ceci a son importance, car si le roi est le représentant de Dieu sur terre en matière de jugement, le magistrat désigné par lui pour exercer la justice à sa place bénéficie par là, lui aussi, au second degré, d'une délégation divine, et l'on pourrait dès lors considérer que tous les actes commis ultérieurement par le Maréchal sont couverts par cette délégation du pouvoir judiciaire. On pourrait même y voir une garantie suffisante pour expliquer qu'au Jugement dernier (celui de la pièce, bien entendu), Hiéronimo mérite l'épithète de «good» qui lui est décernée par le fantôme d'Andréa, et rejoigne les victimes aux Champs Elysées plutôt que les criminels dans les profondeurs du Tartare : mais le légalisme de Kyd (non plus, d'ailleurs, que celui de son héros) ne saurait se satisfaire à si bon compte.

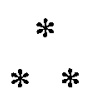

Au-dessus de la simple justice humaine, qui ne pouvait s'exercer, au-dessus de la justice royale, qui a été déniée, il existe une dernière instance, la justice ou «vengeance» divine, vers laquelle se tourner, ce que ne manque pas de faire Hiéronimo dès la scène suivante, dans un long soliloque initial (III. xiii . 1-45), dont les implications profondes me semblent n'avoir guère été clairement perçues.Ce passage souvent jugé incohérent (mais Hiéronimo n'est-il pas alors en proie à un accès de folie?), est un curieux exemple de syncrétisme pagano-chrétien, où se côtoient Sénèque et les Ecritures. Il commence par une citation ( "Vindicta mihi!») qui peut aussi bien provenir de l'Octavie de Sénèque que de l'Epître aux Romains ${ }^{9}$, et se poursuit, après l'affirmation qu'il faut s'en remettre au juge suprême pour le châtiment et son heure :

Ay, heavens will be reveng'd of every ill

Nor will they suffer murder unrepaid;

Then stay, Hieronimo, attend their will, 
For mortal men may not appoint their time

(III. xiii . 2-5)

par des réflexions stoïciennes assez décousues aboutissant à la paradoxale décision :

And to conclude, I will revenge his death!

(ibid. 20), et à la minutieuse description d'un modus operandi de la vengeance fondé sur l'attente et sur la dissimulation (21-45). Il y a ici d'apparentes ruptures logiques qui plaident en faveur de la démence, excuse elle-même d'une vengeance personnelle ainsi assumée. Tout s'éclaire pourtant si l'on veut bien essayer d'envisager l'ensemble de la tirade comme un syllogisme dilué, dont la prémisse mineure serait sousentendue comme allant de soi, fût-ce par référence implicite au passage des Nombres cité plus haut en note ${ }^{10}$. On peut en effet lire alors : «la vengeance et son heure n'appartiennent qu'au Ciel. (Or, je suis l'instrument légitime de cette vengeance). Donc, je me vengerai quand l'heure fixée par le Ciel sera venue.» Cette lecture, si l'on admet l'ellipse suggérée dans le syllogisme d'Hiéronimo, est parfaitement cohérente, et confirmée d'ailleurs par le fait qu'il attende un signe du Ciel pour décider enfin de passer à l'action.

Ce signe, il croira le voir dans la révélation que Bélimpéria est animée à son tour de l'esprit de vengeance (vengeance primaire et instinctive) qui lui a jusqu'à présent fait défaut à lui, Hiéronimo, apôtre de la justice :

Why then, I see that heaven applies our drift, And all the saints do sit soliciting

For vengeance on those cursed murderers

(IV.i. 32-4)

L'attentisme dont il a fait preuve, sa feinte réconciliation avec Lorenzo et la Cour, ne témoignent donc que de son désir de respecter la décision divine, au service de laquelle il s'est mis, et dont, à tort ou à raison, il se croit l'exécutant élu. Là est peut-être la folie, dans l'interprétation du signe céleste qui doit autoriser ses gestes meurtriers, car il n'existe plus aucun code, aucun critère légal sur lesquels fonder l'exécution d'une sentence, même perçue comme légitime. Un message du Ciel, beaucoup moins ambigu pour un esprit sain, lui eût sans doute été fourni par le suicide de sa femme Isabella, rendue folle à son tour par le chagrin et le désespoir de le voir si lent à passer à l'action. D'un autre côté, c'eût 
été, eu égard au dessein de Kyd qui me semble être d'affirmer la prééminence de la justice, affaiblir sa position de jugejusticier désireux, au point d'en perdre la raison, de demeurer l'instrument de l'équité plutôt que de la vengeance personnelle, car la pureté de sa résolution première eût alors été compromise par la prise en compte de ce second coup du sort, et son image ultime eût risqué de n'être plus que celle du vengeur pur et simple, au lieu de celle du jugejusticier qu'il a la conviction d'être, et qui est celle que l'auteur souhaite donner de lui à une partie au moins de son public, pour laquelle le spectacle peut être un objet de réflexion autant que de divertissement. Aussi le suicide d'Isabella n'interviendra-t-il judicieusement qu'une fois la décision prise, et le plan d'exécution mis en route.

Hiéronimo justicier, c'est-à-dire à la fois juge et bourreau, n'agit donc finalement qu'avec l'assurance, fallacieuse peut-être, mais n'oublions pas que sa raison est ébranlée, qu'il est de bout en bout l'agent de la justice la plus haute et la plus stricte. Il a renoncé à exercer ses fonctions officielles dans un cas qui excédait sa compêtence, et qui le faisait à la fois juge et partie. La juridiction royale, vers laquelle il s'était naturellement tourné, a été sourde à son appel, mais ne l'en a pas moins maintenu dans des fonctions dont il s'était démis à la suite de ce qu'il considérait à bon droit comme un déni de justice. Il eût pu voir là un signe que le Ciel l'encourageait à passer outre au fait qu'il était à la fois juge et partie afin que justice fût faite, mais il n'en fait rien tel est son légalisme. Devenu à ses propres yeux l'agent de la justice divine, à défaut de la médiation royale souhaitée, mais avec ce qu'il pense être l'assentiment de celle-ci,puisqu'il a été contre son gré maintenu dans sa charge par la décision même du souverain qui a refusé de l'entendre, il ne consentira pas à agir avant d'avoir confirmation de sa mission par un autre message céleste. L'erreur est peut-être sur ce message, et sur le fait que sa situation de juge et partie n'est explicitement évoquée en aucune occasion, mais le moins que l'on puisse dire est qu'il n'agit jamais sans avoir conscience que la justice (humaine, royale ou divine) est de son côté, et qu'il bénéficie donc largement de circonstances atténuantes si, convaincu d'être en fin de compte le bras même de la justice, il commet des crimes en son nom ${ }^{11}$. Ceci me semble confirmé par le fait que, quels que soient 
les griefs qu'il puisse nourrir contre le roi, il ne songe pas un instant à attenter à sa vie, car, s'il est incontestablement le grand responsable de l'échec de la justice en ce qui le concerne, il demeure aussi pour lui l'indispensable intermédiaire entre la justice divine et la justice humaine, et le garant, puisqu'il continue de lui donner sa caution en le maintenant dans une charge dont il voulait se démettre, de la légitimité de ses décisions judiciaires ${ }^{12}$.

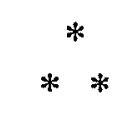

Cela dit, il y a dans la pièce de Kyd d'autres indications, moins techniques sans doute, mais tout aussi significatives, du fait qu'elle est, dans son ensemble, axée sur le problème de la justice de façon beaucoup plus délibérée que sur celui de la vengeance. Son dénouement, par l'intermédiaire de la tragédie de Soliman and Perseda, apparaît comme un des exemples les plus purs qui existent, dans le théâtre élisabéthain, de la justice poétique. Chacun y est en effet puni par où il a péché. Balthazar y est tué par Bélimpéria, pour avoir, en participant au meurtre d'Horatio, tenté de la soustraire à son profit à l'amour de ce dernier, mais aussi pour l'avoir, en un premier temps, frustrée de ses amours sincères, bien qu'illégitimes selon le code de la Cour, avec Don Andréa. Mais l'illustration la plus raffinée de la justice poétique est fournie, me semble-t-il, dans la pièce dans la pièce, par la remarquable inversion des rôles qui fait jouer à Lorenzo le personnage même qui avait été celui d'Horatio (et peutêtre même d'Andréa) dans la réalité : celui du mari ou de l'amant gênant que l'on n'hésite pas à éliminer brutalement afin de favoriser les desseins sur sa belle d'un personnage plus puissant que lui, la raison d'Etat ne s'embarrassant guère des raisons du cœur.

On pourrait sans doute épiloguer aussi sur l'autojustice stoïcienne du double suicide de Bélimpéria et d'Hiéronimo, criminels l'un et l'autre aux yeux de la justice royale, mais que leurs motivations ne sauraient du moins aux yeux du public, rendre passibles d'un châtiment vulgaire. Mais il me paraît plus intéressant de conclure sur l'acte de justice, distributive cette fois, qui préside, sur le plan surnaturel, à la fin de la tragédie. Dans la dernière scène de l'acte 
IV, le fantôme d'Andréa, devenu juge à son tour, assigne à chacune des victimes du dénouement tragique la place qui lui revient dans l'au-delà, aux Champs Elysées ou dans le Tartare, en fonction de ce qu'il estime être leurs mérites ou leurs crimes. La justice divine, ici incarnée, grâce à un transfert mythologique de pure convention, par Andréa assisté de la Vengeance (forme biblique, faut-il le rappeler, du châtiment céleste), se manifeste donc pleinement au terme d'un cheminement tortueux et ténébreux qu'Andréa lui-même a eu beaucoup de peine à suivre et à comprendre. La Vengeance, déléguée par Pluton auprès d'Andréa sur les instances de Proserpine, afin d'autoriser et de sanctionner ses décisions à la suite de ces événements dans le monde des hommes dont ils ont été conjointement spectateurs, peut ainsi légitimement figurer, dans une perspective chrétienne, comme l'agent de cette Providence divine dont les voies sont impénétrables, et le classement d'Hiéronimo parmi les «bons» malgré ses crimes et son suicide tend donc à prouver qu'il n'a été de bout en bout quel'incarnation même de la justice.

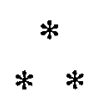

Si l'on considère en conclusion l'abondante postérité que The Spanish Tragedy connut jusqu'à la fermeture des théâtres en 1642, on ne peut que constater, sauf peut-être dans le cas d'Hamlet, où le justicier-vengeur (car il ne s'agit, d'ores et déjà, plus de juge-justicier) ne passe à l'acte que lorsqu'il est lui-même déjà virtuellement mort, victime des machinations de l'objet de sa légitime vengeance, une dégradation croissante du thème. Kyd avait voulu, d'une façon assez claire, me semble-t-il, poser les problèmes en termes stricts de justice, ce que sa maladresse, d'ailleurs toute relative, d'innovateur ne fait peut-être pas assez bien ressortir (sans doute à cause de l'ambiguité de la notion de «vengeance») aux yeux de qui ne s'intéresse qu'à l'action de sa tragédie, suffisamment riche en événements et en coups de théâtre pour anesthésier la réflexion. Je tiens pour ma part que toutes les tragédies de vengeance postérieures à The Spanish Tragedy, quelle que soit leur dette, avouée ou non, envers elle, et le degré auquel elles pensent s'en inspirer, 
ne sont que perversion du modèle kydien, qui ne vise à poser qu'un problème de justice, et je serais donc tenté d'affirmer qu'une des traditions les plus riches du théâtre élisabéthain n'est en fin de compte que le fruit, estimable certes, mais dévoyé, d'un malentendu général sur les intentions premières du modèle.

Jean FUZIER

\section{NOTES}

1. Voir J.M. Maguin : La nuit dans le théâtre de Shakespeare et de ses prédécesseurs, Lille III, 1980, pp. 338-370.

2. Voir Jean Fuzier : «La tragédie de Vengeance élisabéthaine et le théâtre dans le théâtre,» Revue des sciences humaines, N.S., Fasc. 145 (janviermars 1972), 17-33.

3. Un simple coup d'œil à A. Cruden : A Complete Concordance to the Holy Scriptures, London, 1738 (rubriques «vengeance,》 《revenge,》 《justice» «equity,» et mots de la même famille) suffit à s'en assurer.

4. Voir Nombres: XXXV, 19 : «C'est le vengeur du sang qui mettra à mort le meurtrier. Quand il le rencontrera, il le mettra à mort». Le vengeur du sang est le plus proche parent de la victime, le goèl hébreu.

5. Dans "The Spanish Tragedy, or Babylon Revisited,» ds. Essays on Shakespeare and Elizabethan Drama in Honor of Hardin Craig. Edited by Richard Hosley. University of Missouri Press, Columbia, 1962, pp. 23-36.

6. Acte III, scène vi . Le texte de référence est ici The Spanish Tragedy. Edited by P.N. Edwards. The Revels Plays, Methuen \& Co., London, 1959.

7. Nombres XXXV, 30. Voir aussi Jean Fuzier : "Thomas Kyd et L'éthique du spectacle populaire,» Les Langues Modernes no. 4 (juillet-août 1965), 43-50.

8. Le rapprochement s'impose d'autant plus que l'épisode d'Alexandro (III. i) précède strictement la découverte par Hiéronimo de la lettre de Bélimpéría (III. ii), et l'épisode de Pedringano (III. ii-vii).

9. «Haec vindicta debetur mihi?» (Sénc̀que, Octavie, 849), ou «Mihi vindicta» (Rom. XII, 19)? Si le livre qu'Hiéronimo tient en main à son entrée en scène est sans doute un Sénèque (mais le philosophe plutôt que le tragique, au vu de son argumentation ultérieure), la référence biblique,paraphra se donnée par Paul de Deut. XXXII, 35, et reprise d'ailleurs dans Heb. X, 30, semble ici fournir une source plus vraisemblable étant donné le contexte. Voir aussi $P_{s}$. XCIV (XCIII Vulg.) pour le sens général. 
10. Voir note 4 ci-dessus.

11. L'acte le plus contestable d'Hiéronimo est le meurtre du duc de Castille, car ce dernier ne semble avoir d'autre torts que d'être le père de Lorenzo, et peut-être de faire obstacle au suicide du Maréchal. D'un autre côté, on peut remarquer que le duc de Castille est le premier des coupables que la justice d'Andréa voue aux supplices du Tartare. I1 y a donc ici un mystère mal résolu (voir William Empson : "The Spanish Tragedy,» dans Elizabethan Drama : Modern Essays in Criticism. Edited by R.J. Kaufmann, Oxford University Press, New York, 1961, pp. 60-80).

12. Quelques versets après Mihi vindicta, on trouve dans l'Epittre aux Romains le passage suivant, concernant le magistrat : Dei enim minister est tibi in bonum. Si autem malum feceris, time; non enim sine causa gladium portat. Si enim minister est, vindex in iram ei qui malum agit» (XIII, 4). La proximité des deux textes n'est pas sans suggérer que Kyd, citant le premier, ait pu faire son profit de la leçon du second. 